\title{
A different form of acute liver failure
}

\begin{abstract}
Acute liver failure (ALF) is a syndrome characterized by a sudden insult with liver dysfunction and necrosis. It has several etiologies, and it is characterized by an abrupt increase in transaminases, encephalopathy and coagulopathy. The authors intent to expose a rare case of occult neoplasm that caused ALF as the first manifestation and alert the clinicians about the warning signs of ALF as well as the diagnostic approach to be adopted. We present the case of a 71-year-old man that was admitted to hospital due to heart failure and hepatic cytocolestasis, with evolution into hyperacute liver failure on the 7th day of hospitalization, and a final diagnosis of grade 3 small cell neuroendocrine carcinoma.
\end{abstract}

Keywords: acute liver failure, liver biopsy, neuroendocrine carcinoma, occult neoplasm
Volume 12 Issue I - 202I

\author{
Sara Joana Faria, Joana Dias Antunes, Patrícia \\ Amaral, Luísa Loureiro, Isabel Bessa, Amélia \\ Pereira \\ Department of Internal Medicine, Figueira da Foz District \\ Hospital, Portugal
}

\begin{abstract}
Correspondence: Sara Joana Faria, Internal Medicine's Department of Figueira da Foz District Hospital, Rot. do hospital, 3094-001, São Pedro, Figueira da Foz, Coimbra, Portugal,Tel 23340200,Email sarajoanafaria@gmail.com
\end{abstract}

Received: December 12, 2020 | Published: January 25, 202 |
Abbreviations: ALF, acute liver failure; INR, international normalized ratio; ON, occult neoplasm; CT, computed tomography; NEN, neuroendocrine neoplasms

\section{Introduction}

Acute liver failure (ALF) is a rare syndrome, with an estimated incidence of 1 case per 1 million/year and an overall mortality rate of $30-50 \%,{ }^{1-3}$ varying with the cause of liver damage. It is characterized by acute liver injury with subsequent necrosis, encephalopathy, coagulopathy (prolonged prothrombin time/International Normalized Ratio [INR] >1.5) and, in some cases, multiorgan dysfunction.

O'Grady divides ALF into 3 types, according to the onset of encephalopathy: 1) Hyperacute (encephalopathy occurs 7 days after the onset of symptoms); 2) Acute (encephalopathy onset happens 8 to 28days after symptoms/liver damage); 3) Subacute (encephalopathy starts within 28days of the first symptoms). These subcategories are associated with the prognosis, but in reality, they also reflect the underlying causes, which are the true prognostic determinants in ALF. $^{3}$

The most frequent causes of ALF include acetaminophen toxicity and other drugs (DILI - Drug induced Liver Injury), viral hepatitis, autoimmune hepatitis, ischemic liver disease, Wilson's disease, BuddChiari syndrome and HELLP syndrome (hemolysis, elevated liver enzymes, low platelets). Other less frequent causes (6\% according to the US Adult Acute Liver Failure Study Group Registry) include malignancy. The most frequent causes of malignancy with hepatic involvement and progression to ALF are breast carcinoma, small cell lung cancer, lymphoma, melanoma and myeloma. ${ }^{1-4}$

The following article intends to highlight a clinical case of a rare cause of ALF in a patient with Occult Neoplasm (ON).

\section{Description of the clinical case}

A 71-year-old man was admitted to the Emergency Department with dyspnea and orthopnea with 15days of evolution. He had personal history of chronic heart disease, dyslipidemia, active smoking (100units/year) and chronic alcoholism (120grams/day). He denied constitutional symptoms such as weight loss, fever or anorexia. There was no history of drugs or herbal products consumption or recent trips to foreign countries.
At admission, he presented with hemodynamic stability, pulmonary bibasal crackles and edema of the lower limbs, discomfort on palpation of the right flank of the abdomen but without peritoneal irritation. No signs of abdominal collateral circulation or ascitic wave were noted.

The laboratory screening at admission (day 0) revealed: liver cytocolestasis pattern, normal International Normalized Ratio and albumin; amylase of 4364U/L but normal lipase; low level of C-reactive protein $(8.91 \mathrm{mg} / \mathrm{L})$ and an elevated pro b-type natriuretic peptide $(6.108 \mathrm{pg} / \mathrm{mL})$ (Table 1). Arterial blood gas analysis revealed hypoxemia $(\mathrm{PaO} 250.9 \mathrm{mmHg}$ ) without acidosis or lactacidemia. The chest X-Ray demonstrated signs of cardiomegaly and mild bilateral pleural effusion. Abdominal ultrasound (US) revealed dysmorphic liver with diffuse pericentrimetric nodular pattern, suggestive of chronic liver disease. The patient was admitted to the Medical Care Unit with the diagnosis of decompensated chronic heart failure and elevated liver cytocolestase parameters (not yet fully clarified).

Table I Analytical control

\begin{tabular}{lllll}
\hline \multirow{2}{*}{$\begin{array}{l}\text { Laboratory } \\
\text { parameters }\end{array}$} & \multicolumn{4}{c}{ Alterations during hospitalization (days) } \\
\cline { 2 - 5 } & $\mathbf{0}$ & 4th & 6th & 7th \\
\hline AST (U/L) & 283 & 422 & 686 & 2752 \\
ALT (U/L) & 395 & 446 & 1027 & 1636 \\
LDH (U/L) & 7065 & 13848 & 21658 & 24848 \\
AP (U/L) & 292 & 1348 & 571 & 612 \\
GGT (U/L) & 1506 & 1603 & 1603 & 1591 \\
TB (mg/dL) & 1.72 & 3.15 & 5.34 & 7.25 \\
DB (mg/dL) & 1.22 & 2.48 & 4.99 & 6.46 \\
INR & 1 & 1.1 & 1.2 & 1.6 \\
Albumin (g/dl) & 4 & 4 & 3.4 & 3 \\
\hline
\end{tabular}

ALT, alanine aminotransferase; AP, alkaline phosphatase; AST, aspartate aminotransferase; DB, direct bilirubin; GGT, gamma-glutamyl transpeptidase; INR, international normalized ratio; $L D H$, lactate dehydrogenase; TB, total bilirubin 
Subsequent complementary study demonstrated negative serologies for hepatotropic viruses. Immunological markers for autoimmune hepatitis were absent. Serum ferritin and urinary copper assay were within normal values. Alpha-Fetoprotein, ProstateSpecific Antigen and 72-4 Cancer Antigen were also normal. There was an elevation of Carcinoembryonic Antigen (CEA: 49.8ng/ml) and Cancer Antigen 19-9 (CA 19-9: 975.70U/ml).

Whole body-CT (Computed Tomography) demonstrated important cardiomegaly, a small pleural effusion, several hepatic hypodense micronodules, and a larger nodule (of 5 centimeters) located in the left lobe. There was no mention of hepatosplenomegaly (Figure 1). CT-guided liver biopsy was performed.

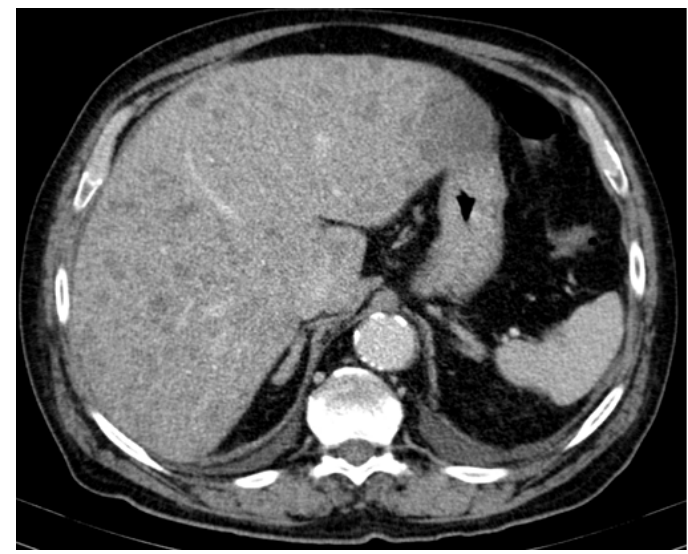

Figure I CT- scan: Multiple hypodense liver nodules and a larger one in the left lobe of the liver.

Throughout hospitalization, patient got worse, especially on the $4^{\text {th }}$ day after admission, with grade II encephalopathy and progressive increase in hepatic cytocolestasis parameters, as shown in Table 1. The possibility of transferring to a Center with Intensive Care Unit was discussed and refused by the family.

On the 7th day, the patient developed ALF (Table 1) with subsequent multiorgan dysfunction. He died the next day (8th day). Later, the biopsy result concluded the diagnosis of liver involvement by grade 3 small cell neuroendocrine carcinoma (Figures $2 \& 3$ ).

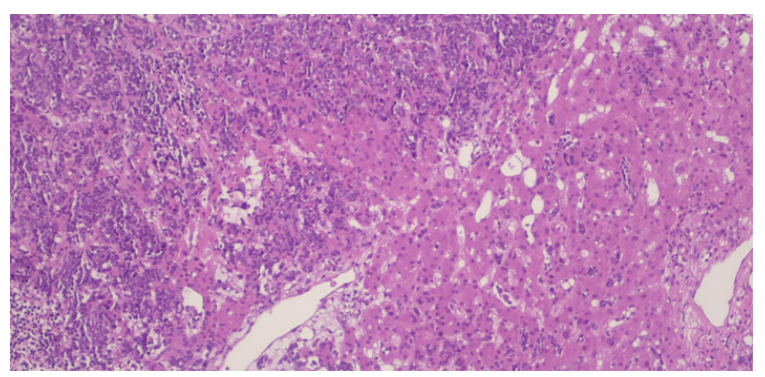

Figure 2 Infiltration of the liver by neoplastic cells (small, round and blue) with extensive areas of necrosis (Hematoxylin \& Eosin staining; 40x).

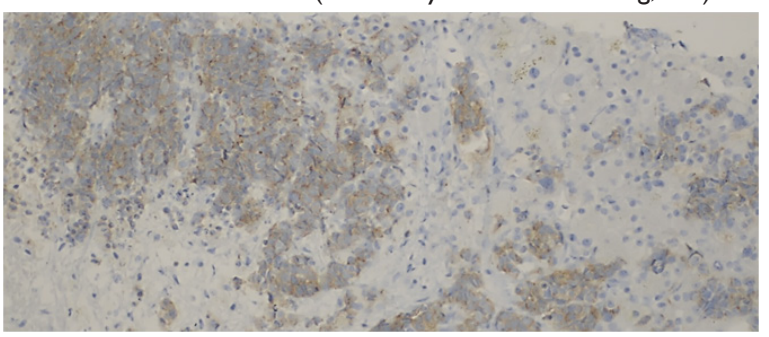

Figure 3 Immunohistochemical staining - tumor cells with synaptophysin expression $(200 x)$.

\section{Discussion}

Neuroendocrine neoplasms (NEN) are part of a heterogeneous group of diseases, with great variation in biological behavior and histological diagnosis. The prognosis is strongly correlated with histological differentiation and the proliferation rate (G1low grade; G2-intermediate grade; G3-high grade). ${ }^{5-7}$ Most NENs are characterized by slow growth and secretion of vasoactive or hormonal substances. Other NENs are highly aggressive and classified as neuroendocrine carcinomas of large or small cells (poorly differentiated; high grade proliferation rate [G3]). ${ }^{6-8}$

Occult neoplasms $(\mathrm{ON})$ constitute $4-5 \%$ of all invasive neoplasms and within these group the NEN represent less than 5\%. The diagnosis of NEN as ON is possible in most cases due to symptoms related to metastases $^{5,9}$ and neuroendocrine carcinomas, although rare, usually manifest rapidly and aggressively. In our case, the manifestations related to metastases ended up being masked initially by a multitude of factors including the right heart clinical failure. However, in a few days the patient developed hyperacute liver failure that evolved negatively, shortening the time of etiological investigation and treatment.

In fact, the first clinical manifestations of ALF may be nonspecific (anorexia, fatigue, abdominal pain or fever). Nevertheless, when liver function worsens significantly, it progresses invariably with hepatic encephalopathy, coagulopathy, and hemodynamic instability with multiple organ dysfunction which are common features in all forms of ALF. ${ }^{4,10}$ Hyperacute liver failure evolves with hepatic encephalopathy that often precedes jaundice, as well as a significant increase in transaminases and a slight increase in bilirubin. Cerebral edema and intracranial hypertension with risk of herniation are very common and the cause of death. ${ }^{1-5}$

The initial presentation of our patient showed some confounding factors - clinical presentation on admission as decompensated heart failure, absence of constitutional symptoms, heavy drinking and description of nodular pattern on US suggesting liver disease of alcoholic etiology. Nevertheless, the rapidly progressive degradation and the exclusion of more frequent causes of ALF guided us to metastatic infiltration. The performance of liver biopsy was crucial for the final diagnosis and identification of the specific etiology of ALFG3 small cell neuroendocrine carcinoma. After the diagnosis that was post-mortem, we were able to understand that the final outcome predictable by hyperacute ALF was due to this aggressive character of the carcinoma. It was not possible for us to have time to investigate the primary neoplasm.

\section{Conclusion}

ALF has several causes - toxic, viral, autoimmune, ischemic, metabolic, malignant, among others. Whenever a patient presents with acute liver injury, encephalopathy, and coagulopathy, all hypothesis must be raised. A quick diagnostic approach and therapeutic intervention can mean a change in prognosis. Some causes, as in our patient, invariably have a negative outcome, but at least we reached a final diagnosis by liver biopsy, despite being post-mortem. ${ }^{1-5,10}$

\section{Acknowledgments}

None.

\section{Conflicts of interest}

The authors declare that there are no conflicts of interest. 


\section{Funding}

None.

\section{References}

1. Stravitz R, Lee W. Acute Liver Failure. Lancet. 2019;394 (10201):869881.

2. Oketani M, Ido A, Tsubouchi H. Changing etiologies and outcomes of acute liver failure: A perspective from Japan. J Gastroenterol Hepatol. 2011;26 Suppl 1:65-71.

3. Grady, JGO. Acute liver failure. Postgrad Med J. 2005;81(953):148-154.

4. Grek A, Arasi L. Acute Liver Failure. $A A C N$ Adv Crit Care. 2016;27(4):420-429.

5. Spigel DR, Hainsworth JD, Greco FA. Neuroendocrine Carcinoma of Unknown Primary Site. Semin Oncol. 2009;36(1):52-59.
6. Klimstra DS, Kloppell G, La Rosa S, et al. Classification of neuroendocrine neoplasms of the digestive system. In: WHO Classification of Tumours: Digestive System Tumours, 5th edn. WHO Classification of Tumours Editorial Board. Lyon: International Agency for Research on Cancer; 2019. p. 16

7. Strosberg J, Halfdanarson T, Bellizi M, et al. The North American Neuroendocrine Tumor Society Consensus Guidelines for Surveillance and Medical Management of Midgut Neuroendocrine Tumors. Pancreas Journal. 2017;46(6):707-714.

8. Kunz PL. Carcinoid and Neuroendocrine Tumors: Building on Success. $J$ Clin Oncol. 2015;33(16):1855-1863.

9. Greco F, Hainsworth J. Cancer of unknown primary site. Cancer: Principles and Practice of Oncology. 10 ${ }^{\text {th }}$ edn. In: DeVita VT Jr, Hellman S, Rosenberg SA, Editors. USA, Philadelphia: Wolters Kluwer; 2015. p. 1720 .

10. Wang D, Yin Y, Yao Y. Advances in the management of acute liver failure. World Journal of Gastroenterology. 2013;19(41):7069-7077. 